\title{
Gemcitabine/Cisplatin/Everolimus Regimen
}

National Cancer Institute

\section{Source}

National Cancer Institute. Gemcitabine/Cisplatin/Everolimus Regimen. NCI Thesaurus.

Code C156266.

A regimen consisting of gemcitabine, cisplatin and everolimus that may be used for the treatment of a variety of cancers, such as urothelial, bladder and breast cancer. 